\title{
IUCrJ
}

Volume 2 (2015)

Supporting information for article:

Combinatorial selection of molecular conformations and supramolecular synthons in quercetin cocrystal landscapes. A route to ternary solids

Ritesh Dubey and Gautam R. Desiraju 


\section{Combinatorial selection of molecular conformations and supramolecular synthons in quercetin cocrystal landscapes. A route to ternary solids}

Ritesh Dubey and Gautam R. Desiraju*

Solid State and Structural Chemistry Unit, Indianstitute of Science, Bangalore 560 012, India

1. Virtual library of molecular conformations

2. Coformers used in the studies

3. Experimental details

4. Normalized hydrogen bond distances

5. Crystallographic tables of the experimental structures

S1. Virtual library of molecular conformations--- M062x/6-31++g(d,p) level calculation

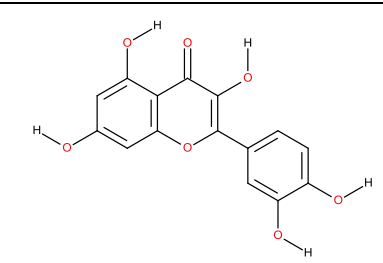

Conf $1 \mathrm{~A}$

$0.84 \mathrm{kcal} / \mathrm{mol}$

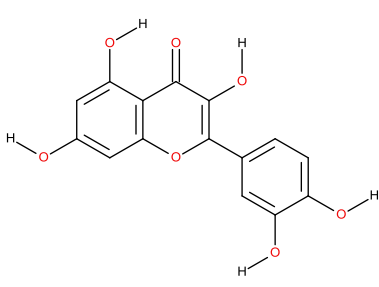

Conf $3 \mathrm{~A}$

$4.98 \mathrm{kcal} / \mathrm{mol}$

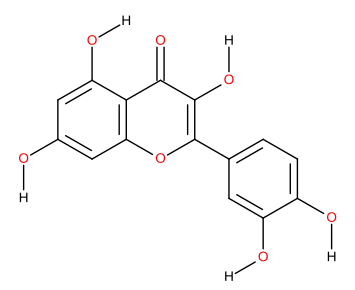

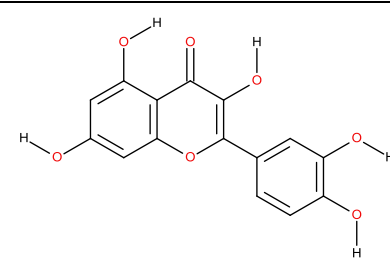

Conf 1B

$1.09 \mathrm{kcal} / \mathrm{mol}$

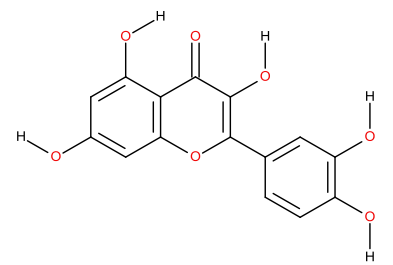

Conf 3B

$4.63 \mathrm{kcal} / \mathrm{mol}$

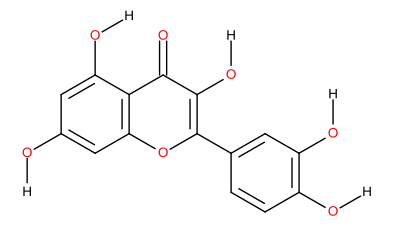

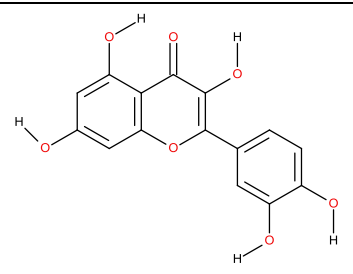

Conf 2A

$0.63 \mathrm{kcal} / \mathrm{mol}$

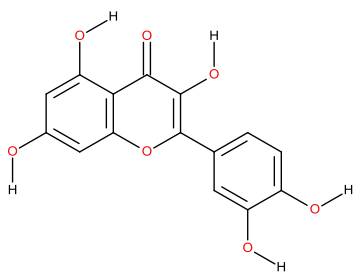

Conf 5A

$1.08 \mathrm{kcal} / \mathrm{mol}$

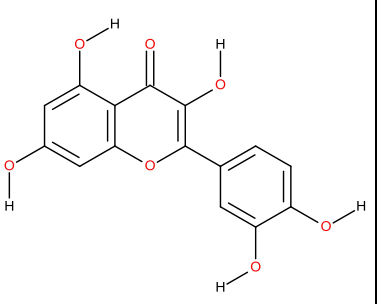

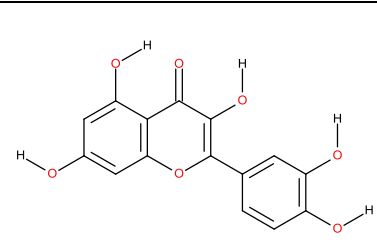

Conf 2B

$0.00 \mathrm{kcal} / \mathrm{mol}$<smiles>CCc1cc(I)cc2cc(-c3ccc(I)c(OC)c3)c(CI)c(C)c12</smiles>

Conf 5B

$1.55 \mathrm{kcal} / \mathrm{mol}$

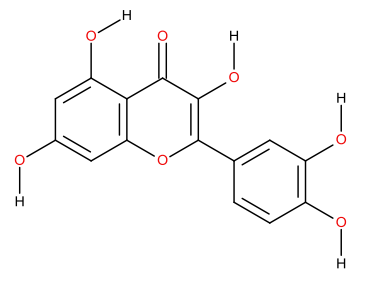




\begin{tabular}{c|c|c|c}
\hline Conf 6A & Conf 6B & Conf $7 \mathrm{~A}$ & Conf 7B \\
$1.27 \mathrm{kcal} / \mathrm{mol}$ & $0.36 \mathrm{kcal} / \mathrm{mol}$ & $5.51 \mathrm{kcal} / \mathrm{mol}$ & $5.09 \mathrm{kcal} / \mathrm{mol}$ \\
& & & \\
\hline
\end{tabular}

S2. Coformers used in the study

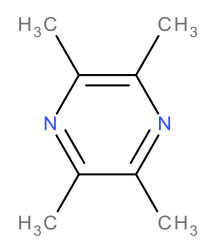

TMP<smiles>c1cc(-c2ccncc2)ccn1</smiles>

44BP

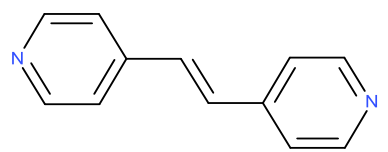

DPE-I<smiles>c1ccc2nc3ccccc3nc2c1</smiles>

PHE

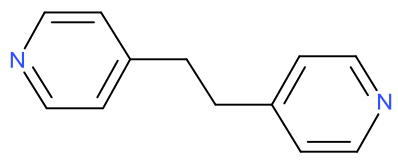

DPE-II

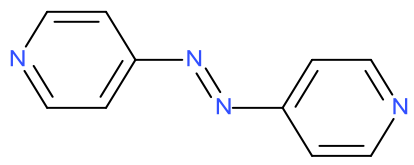

44AP

\section{S3. Experimental details}

a) QUE:TMP (Form I): Yellow plate shaped crystals were grown from (1:1) stoichiometric ratios of the compounds using DMSO as a solvent after fifteen days.

b) QUE:TMP (Form II): Yellow plate shaped crystals were grown from (2:1) stoichiometric ratios of the compounds using DMF as a solvent after ten days.

c) QUE:TMP (Form III): Yellow thin plate shaped crystals were grown from $(3: 1)$ stoichiometric ratios of the compounds using 1,4-dioxane as a solvent after six days.

d) $Q U E: T M P$ (Form IV): Yellow thin plate shaped crystals were grown from (3:1) stoichiometric ratios of the compounds using THF solvent after three days. 
e) $Q U E: 44 B P($ Form I): Yellow thin plate shaped crystals were obtained from $(1: 1)$ stoichiometric ratios of the compounds using 1,4-dioxane as solvent after five-six days.

f) $Q U E: 44 B P$ (Form II):Yellow thin plate shaped crystals were grown from (1:1) stoichiometric ratios of the compounds using THF solvent after three days.

g) $Q U E: 44 B P$ (Form III): Yellow plate shaped crystals were grown from (1:1) stoichiometric ratios of the compounds using DMF solvent after ten days.

h) $Q U E: 44 B P($ Form IV): Monohydrate yellow plate shaped crystals were grown from (1:4) stoichiometric ratios of the compounds using DMSO solvent after ten to fifteen days.

i) QUE:DPE-I (Form I): Yellow plate shaped crystals were grown from (1:1) stoichiometric ratios of the compounds using mixture of 1,4-dioxane- $\mathrm{MeOH}$ solvents after six days.

j) QUE:DPE-I (Form II): Yellow plate shaped crystals were grown from (1:1) stoichiometric ratios of the compounds using THF solvent after three days.

k) QUE:DPE-I (Form III):Yellow plate shaped crystals were grown from (2:1) stoichiometric ratios of the compounds using DMF solvent after six days.

1) QUE:DPE-I (Form IV): Yellow plate shaped crystals were grown from (1:1) stoichiometric ratios of the compounds using DMSO solvent, after twelve to fifteen days.

m) QUE:DPE-I (Form V): We utilized the liquid diffusion technique where, the $(1: 1)$ solution of QUE:DPE-I was layered with toluene solution of biphenyl. Yellow-red plate shaped diffraction quality crystals were obtained after ten to twelve days. 
n) QUE:DPE-II (Form I): Yellow plate shaped crystals were grown from (3:2) stoichiometric ratios of the compounds using mixture of THF-MeOH solvents after four days.

o) QUE:DPE-II (Form II): Yellow plate shaped crystals were grown from (1:1) stoichiometric ratios of the compounds using DMF solvent after ten days.

p) QUE:DPE-II (Form III): Yellow plate shaped crystals were grown from (2:1) stoichiometric ratios of the compounds using DMF solvent after ten to twelve days.

q) $Q U E: 44 A P$ (Form I): Red plate shaped crystals were grown from (2:1) stoichiometric ratios of the compounds using THF solvent after four days.

r) QUE:44AP (Form II): Red plate shaped crystals were grown from (2:1) stoichiometric ratios of the compounds using DMF solvent after ten days.

s) QUE:PHE (Form I): Red plate shaped crystals were grown from (1:1:1) QUE:PHE:ANT stoichiometric ratios using 1,4-dioxane solvents after seven days.

t) QUE:PHE (Form II): Yellow-red plate shaped crystals were grown from (1:1) stoichiometric ratios of the compounds using mixture of $\mathrm{CH}_{3} \mathrm{CN}: \mathrm{MeOH}$ solvents after four days.

u) QUE:PHE (Form III): Red plate shaped crystals were grown from (1:2) stoichiometric ratios of the compounds using $\mathrm{MeOH}$ solvent after four to five days.

v) QUE:PHE (Form IV): Red plate shaped crystals were grown from (1:1) QUE:PHE stoichiometric ratios using mixture of $\mathrm{CH}_{3} \mathrm{CN}: \mathrm{MeOH}$ solvents after ten to twelve days. 
w) QUE:PHE (Form V): Yellow-red plate shaped crystals were grown from (1:1:1) QUE:PHE:22TP stoichiometric ratios using $\mathrm{CH}_{3} \mathrm{CN}$ solvent in cold conditions $\left(5^{\circ} \mathrm{C}\right)$ after twelve days.

x) QUE:44BP:22TP: We utilized the liquid diffusion technique where, the (1:1) $i$-PrOH solution of QUE:44BP was layered with toluene solution of 22TP. Yellow-red plate shaped diffraction quality crystals were obtained after twelve to fifteen days.

y) QUE:44BP:TTF: We utilized the liquid diffusion technique where, the (1:1) EtOH solution of QUE:44BP was layered with toluene solution of TTF. Yellow-red plate shaped diffraction quality crystals were obtained after twelve to fifteen days.

z) QUE:DPE-I:22TP: We utilized the liquid diffusion technique where, the (1:1) $i$-PrOH solution of QUE:DPE-I was layered with toluene solution of 22TP. Yellow plate shaped diffraction quality crystals were obtained after twelve to fifteen days.

aa) QUE:DPE-I:PYR: We utilized the liquid diffusion technique where, the (1:1) $i$-PrOH solution of QUE:DPE-I was layered with toluene solution of PYR. Yellow plate shaped diffraction quality crystals were obtained after twelve to fifteen days.

bb) QUE:DPE-I:ANT: We utilized the liquid diffusion technique where, the (1:1) $i$-PrOH solution of QUE:DPE-I was layered with toluene solution of ANT. Yellow plate shaped diffraction quality crystals were obtained after twelve to fifteen days.

\begin{tabular}{|l|l|l|l|l|}
\hline & \multicolumn{1}{|c|}{ Stoichiometric Ratios } & \multicolumn{1}{|c|}{ Solvent } & $\begin{array}{c}\text { Molecular } \\
\text { Conformation }\end{array}$ & \multicolumn{1}{|c|}{$\begin{array}{c}\text { Supramolecular } \\
\text { Synthon }\end{array}$} \\
\hline & & & & \\
\hline QUE:TMP (Form I) & $1: 1$ & DMSO & Conf 6B & Synthon A \\
\hline QUE:TMP (Form II) & $2: 1$ & DMF & Conf 6B & Synthon B \\
\hline QUE:TMP (Form III) & $3: 1$ & 1,4 -Dioxane & Conf 5B & Synthon E \\
\hline
\end{tabular}




\begin{tabular}{|c|c|c|c|c|}
\hline QUE:TMP (Form IV) & $3: 1$ & THF & Conf 5B & Synthon E \\
\hline QUE:44BP (Form I) & $1: 1$ & 1,4-Dioxane & Conf 5B & Synthon E \\
\hline QUE:44BP (Form II) & $1: 1$ & THF & Conf 5B & Synthon E \\
\hline QUE:44BP (Form III) & $1: 1$ & DMF & Conf 5B & Synthon E \\
\hline QUE:44BP (Form IV) & $1: 4$ & DMSO & Conf 7B & Synthon F \\
\hline QUE:DPE-I (Form I) & $1: 1$ & 1,4-Dioxane/MeOH & Conf 5B & Synthon E \\
\hline QUE:DPE-I (Form II) & $1: 1$ & THF & Conf 5B & Synthon E \\
\hline QUE:DPE-I (Form III) & $2: 1$ & DMF & Conf 5B & Synthon E \\
\hline QUE:DPE-I (Form IV) & $1: 1$ & DMSO & Conf 7B & Synthon $\mathrm{G}$ \\
\hline QUE:DPE-I (Form V) & $\begin{array}{l}\text { 1:1, liquid diffusion with } \\
\text { toluene solution of biphenyl }\end{array}$ & $i$-PrOH & Conf 1B & Synthon H \\
\hline QUE:DPE-II (Form I) & $3: 2$ & THF/MeOH & Conf 5B & Synthon E \\
\hline QUE:DPE-II (Form II) & $1: 1$ & DMF & Conf 7B & Synthon G \\
\hline QUE:DPE-II (Form III) & $2: 1$ & DMF & Conf 6B & Synthon C \\
\hline QUE:44AP (Form I) & $2: 1$ & THF & Conf 5B & Synthon E \\
\hline QUE:44AP (Form II) & $2: 1$ & DMF & Conf 1B & Synthon H \\
\hline QUE:PHE (Form I) & 1:1:1 with ANT & 1,4-Dioxane & Conf 5B & Synthon E \\
\hline QUE:PHE (Form II) & $1: 1$ & $\mathrm{CH}_{3} \mathrm{CN} / \mathrm{MeOH}$ & Conf 5B & Synthon E \\
\hline QUE:PHE (Form III) & $1: 2$ & $\mathrm{MeOH}$ & Conf 6B & Synthon D \\
\hline QUE:PHE (Form IV) & $1: 1$ & $\mathrm{CH}_{3} \mathrm{CN} / \mathrm{MeOH}$ & Conf 7B & Synthon G \\
\hline QUE:PHE (Form V) & 1:1:1 with $22 \mathrm{TP}$ & $\mathrm{CH}_{3} \mathrm{CN}\left(5^{\circ} \mathrm{C}\right)$ & Conf 2A & Synthon I \\
\hline QUE:44BP:22TP & $\begin{array}{l}1: 1, \text { liquid diffusion with } \\
\text { toluene solution of } 22 \mathrm{TP}\end{array}$ & $i$-PrOH & Conf 5B & Synthon E \\
\hline QUE:44BP:TTF & $\begin{array}{l}\text { 1:1, liquid diffusion with } \\
\text { toluene solution of TTF }\end{array}$ & $\mathrm{EtOH}$ & Conf 6B & Synthon B \\
\hline QUE:DPE-I:22TP & $\begin{array}{l}1: 1, \text { liquid diffusion with } \\
\text { toluene solution of } 22 \mathrm{TP}\end{array}$ & $i$-PrOH & Conf 5B & Synthon E \\
\hline QUE:DPE-I:ANT & $\begin{array}{l}1: 1, \text { liquid diffusion with } \\
\text { toluene solution of ANT }\end{array}$ & $i$-PrOH & Conf 1A & Synthon J \\
\hline QUE:DPE-I:PYR & $\begin{array}{l}\text { 1:1, liquid diffusion with } \\
\text { toluene solution of PYR }\end{array}$ & $i$-PrOH & Conf 6B & Synthon B \\
\hline
\end{tabular}

Table S1 Normalized hydrogen bond distances

\begin{tabular}{|c|c|c|c|c|c|c|}
\hline Compound & $\mathbf{D}-\mathbf{H} \cdots \mathbf{A}^{\#}$ & $\mathbf{r}(\mathbf{D}-\mathbf{H}) / \AA$ & $\mathbf{r}(\mathbf{D}-\mathbf{A}) / \AA$ & $\mathbf{r}(\mathbf{H} \cdots \mathbf{A}) / \AA$ & $\angle \mathbf{D}-\mathbf{H} \cdots \mathbf{A}^{\mathbf{0}}$ & Symmetry \\
\hline QUE:TMP & $\mathrm{O}(1)-\mathrm{H}(1 \mathrm{O}) \cdots \mathrm{N}(6)$ & 0.98 & $2.636(5)$ & 1.67 & 168 & $\mathrm{x},-1-\mathrm{y}, 1 / 2+\mathrm{z}$ \\
\hline
\end{tabular}




\begin{tabular}{|c|c|c|c|c|c|c|}
\hline \multirow[t]{5}{*}{ Form I } & $\mathrm{O}(2)-\mathrm{H}(2 \mathrm{O}) \cdots \mathrm{N}(5)$ & 0.98 & $2.927(5)$ & 2.32 & $119^{\prime}$ & $1+x,-y, 1 / 2+z$ \\
\hline & $\mathrm{O}(4)-\mathrm{H}(4 \mathrm{O}) \cdots \mathrm{N}(1)$ & 0.98 & $2.811(4)$ & 1.88 & $157^{\prime}$ & $1+\mathrm{x}, 1+\mathrm{y}, \mathrm{z}$ \\
\hline & $\mathrm{O}(5)-\mathrm{H}(5 \mathrm{O}) \cdots \mathrm{N}(3)$ & 0.98 & $2.848(5)$ & 1.87 & 174 & $1+\mathrm{x}, 1+\mathrm{y}, \mathrm{z}$ \\
\hline & $\mathrm{O}(6)-\mathrm{H}(60) \cdots \mathrm{O}(2)$ & 0.98 & $2.732(4)$ & 1.79 & $158^{\prime}$ & $\mathrm{x},-\mathrm{y},-1 / 2+\mathrm{z}$ \\
\hline & $\mathrm{C}(21)-\mathrm{H}(21 \mathrm{~A}) \cdots \mathrm{O}(1)$ & 1.08 & $3.373(5)$ & 2.50 & 137 & $\mathrm{x},-1-\mathrm{y},-1 / 2+\mathrm{z}$ \\
\hline \multirow{6}{*}{$\begin{array}{l}\text { QUE:TMP } \\
\text { Form II }\end{array}$} & $\mathrm{O}(1)-\mathrm{H}(1 \mathrm{O}) \cdots \mathrm{N}(4)$ & 0.98 & $2.692(3)$ & 1.71 & 174 & $\mathrm{x},-1+\mathrm{y}, \mathrm{z}$ \\
\hline & $\mathrm{O}(2)-\mathrm{H}(2 \mathrm{O}) \cdots \mathrm{O}(5)$ & 0.98 & $3.068(3)$ & 2.45 & $121^{\prime}$ & $\mathrm{x}, 1 / 2-\mathrm{y},-1 / 2+\mathrm{z}$ \\
\hline & $\mathrm{O}(4)-\mathrm{H}(4 \mathrm{O}) \cdots \mathrm{N}(2)$ & 0.98 & $2.713(3)$ & 1.75 & $164^{\prime}$ & $2-x, 1-y,-z$ \\
\hline & $\mathrm{O}(5)-\mathrm{H}(5 \mathrm{O}) \cdots \mathrm{N}(1)$ & 0.98 & $2.810(3)$ & 1.83 & 175 & - \\
\hline & $\mathrm{O}(6)-\mathrm{H}(60) \cdots \mathrm{O}(3)$ & 0.98 & $2.787(3)$ & 1.86 & $156^{\prime}$ & $\mathrm{x}, 1 / 2-\mathrm{y}, 1 / 2+\mathrm{z}$ \\
\hline & $\mathrm{C}(21)-\mathrm{H}(21 \mathrm{~A}) \cdots \mathrm{O}(2)$ & 1.08 & $3.318(4)$ & 2.38 & 144 & $\mathrm{x}, 1 / 2-\mathrm{y}, 1 / 2+\mathrm{z}$ \\
\hline \multirow{6}{*}{$\begin{array}{l}\text { QUE:TMP } \\
\text { Form III }\end{array}$} & $\mathrm{O}(1)-\mathrm{H}(1 \mathrm{O}) \cdots \mathrm{N}(2)$ & 0.98 & $2.691(2)$ & 1.71 & 174 & - \\
\hline & $\mathrm{O}(4)-\mathrm{H}(4 \mathrm{O}) \cdots \mathrm{O}(3)$ & 0.98 & $2.6989(17)$ & 1.82 & $147^{\prime}$ & $1-\mathrm{x},-\mathrm{y},-\mathrm{z}$ \\
\hline & $\mathrm{O}(5)-\mathrm{H}(5 \mathrm{O}) \cdots \mathrm{O}(1)$ & 0.98 & $2.7593(17)$ & 1.83 & 157 & $\mathrm{x}, \mathrm{y},-1+\mathrm{z}$ \\
\hline & $\mathrm{O}(6)-\mathrm{H}(6 \mathrm{O}) \cdots \mathrm{N}(1)$ & 0.98 & $2.7723(19)$ & 1.79 & 175 & $-1-x, 1-y,-z$ \\
\hline & $\mathrm{C}(21)-\mathrm{H}(21 \mathrm{~A}) \cdots \mathrm{O}(2)$ & 1.08 & $3.386(2)$ & 2.49 & 140 & $-1+x, 1+y, z$ \\
\hline & $\mathrm{C}(24)-\mathrm{H}(24 \mathrm{~A}) \cdots \mathrm{O}(9)$ & 1.08 & $3.546(4)$ & 2.46 & 178 & $1-\mathrm{x}, 1-\mathrm{y},-\mathrm{z}$ \\
\hline \multirow{6}{*}{$\begin{array}{l}\text { QUE:TMP } \\
\text { Form IV }\end{array}$} & $\mathrm{O}(1)-\mathrm{H}(1 \mathrm{O}) \cdots \mathrm{N}(2)$ & 0.98 & $2.688(3)$ & 1.71 & 172 & $2-x,-y, 1-z$ \\
\hline & $\mathrm{O}(4)-\mathrm{H}(4 \mathrm{O}) \cdots \mathrm{O}(3)$ & 0.98 & $2.677(2)$ & 1.76 & $153^{\prime}$ & $-\mathrm{x}, 1-\mathrm{y}, 1-\mathrm{z}$ \\
\hline & $\mathrm{O}(5)-\mathrm{H}(5 \mathrm{O}) \cdots \mathrm{O}(1)$ & 0.98 & $2.766(2)$ & 1.87 & 149 & $\mathrm{x}, \mathrm{y}, 1+\mathrm{z}$ \\
\hline & $\mathrm{O}(6)-\mathrm{H}(6 \mathrm{O}) \cdots \mathrm{N}(1)$ & 0.98 & $2.765(3)$ & 1.80 & 166 & - \\
\hline & $\mathrm{C}(22)-\mathrm{H}(22 \mathrm{~A}) \cdots \mathrm{O}(2)$ & 1.08 & $3.404(3)$ & 2.49 & 142 & $1-x, 1-y, 1-z$ \\
\hline & $\mathrm{C}(24)-\mathrm{H}(24 \mathrm{~B}) \cdots \mathrm{O}(2)$ & 1.08 & $3.429(4)$ & 2.47 & 146 & $1-x,-y, 1-z$ \\
\hline \multirow{5}{*}{$\begin{array}{c}\text { QUE:44BP } \\
\text { Form I }\end{array}$} & $\mathrm{O}(1)-\mathrm{H}(1 \mathrm{O}) \cdots \mathrm{N}(1)$ & 0.98 & $2.6635(15)$ & 1.69 & 172 & $-\mathrm{x}, 1-\mathrm{y},-\mathrm{z}$ \\
\hline & $\mathrm{O}(4)-\mathrm{H}(4 \mathrm{O}) \cdots \mathrm{O}(3)$ & 0.98 & $2.6750(14)$ & 1.81 & $145^{\prime}$ & $1-x, 1-y,-z$ \\
\hline & $\mathrm{O}(6)-\mathrm{H}(5 \mathrm{O}) \cdots \mathrm{O}(1)$ & 0.98 & $2.7455(12)$ & 1.82 & 156 & $\mathrm{x}, \mathrm{y}, 1+\mathrm{z}$ \\
\hline & $\mathrm{O}(7)-\mathrm{H}(6 \mathrm{O}) \cdots \mathrm{N}(2)$ & 0.98 & $2.6837(15)$ & 1.70 & 174 & $-1+\mathrm{x}, 1+\mathrm{y}, \mathrm{z}$ \\
\hline & $\mathrm{C}(22)-\mathrm{H}(22) \cdots \mathrm{O}(6)$ & 1.08 & $3.2685(16)$ & 2.37 & 139 & $1-x, 1-y, 1-z$ \\
\hline \multirow{5}{*}{$\begin{array}{l}\text { QUE:44BP } \\
\text { Form II }\end{array}$} & $\mathrm{O}(1)-\mathrm{H}(1 \mathrm{O}) \cdots \mathrm{N}(1)$ & 0.98 & $2.647(4)$ & 1.67 & 173 & $-1+\mathrm{x}, \mathrm{y}, \mathrm{z}$ \\
\hline & $\mathrm{O}(4)-\mathrm{H}(4 \mathrm{O}) \cdots \mathrm{O}(3)$ & 0.98 & $2.678(3)$ & 1.80 & $147^{\prime}$ & $1-x, 1-y, 1-z$ \\
\hline & $\mathrm{O}(5)-\mathrm{H}(5 \mathrm{O}) \cdots \mathrm{O}(1)$ & 0.98 & $2.730(3)$ & 1.83 & 151 & $\mathrm{x}, \mathrm{y}, 1+\mathrm{z}$ \\
\hline & $\mathrm{O}(6)-\mathrm{H}(6 \mathrm{O}) \cdots \mathrm{N}(2)$ & 0.98 & $2.697(4)$ & 1.72 & 173 & $-\mathrm{x}, 2-\mathrm{y}, 1-\mathrm{z}$ \\
\hline & $\mathrm{C}(25)-\mathrm{H}(25) \cdots \mathrm{O}(5)$ & 1.08 & $3.250(4)$ & 2.34 & 141 & $\mathrm{x}, \mathrm{y},-1+\mathrm{z}$ \\
\hline \multirow{5}{*}{$\begin{array}{c}\text { QUE:44BP } \\
\text { Form III }\end{array}$} & $\mathrm{O}(1)-\mathrm{H}(1 \mathrm{O}) \cdots \mathrm{N}(1)$ & 0.98 & $2.663(2)$ & 1.68 & 175 & $-1-x, 2-y, 1-z$ \\
\hline & $\mathrm{O}(4)-\mathrm{H}(4 \mathrm{O}) \cdots \mathrm{O}(3)$ & 0.98 & $2.678(2)$ & 1.80 & $147^{\prime}$ & $1-x, 1-y, 1-z$ \\
\hline & $\mathrm{O}(5)-\mathrm{H}(5 \mathrm{O}) \cdots \mathrm{O}(1)$ & 0.98 & $2.753(2)$ & 1.82 & 157 & $\mathrm{x}, \mathrm{y}, 1+\mathrm{z}$ \\
\hline & $\mathrm{O}(6)-\mathrm{H}(6 \mathrm{O}) \cdots \mathrm{N}(2)$ & 0.98 & $2.719(2)$ & 1.74 & 174 & - \\
\hline & $\mathrm{C}(17)-\mathrm{H}(17) \cdots \mathrm{O}(5)$ & 1.08 & $3.300(3)$ & 2.36 & 144 & $-\mathrm{x}, 2-\mathrm{y}, 2-\mathrm{z}$ \\
\hline \multirow{3}{*}{$\begin{array}{l}\text { QUE:44BP } \\
\text { Form IV }\end{array}$} & $\mathrm{O}(1)-\mathrm{H}(1 \mathrm{O}) \cdots \mathrm{N}(5)$ & 0.98 & $2.6631(19)$ & 1.68 & 176 & 1-x,1-y,1-z \\
\hline & $\mathrm{O}(4)-\mathrm{H}(4 \mathrm{O}) \cdots \mathrm{N}(3)$ & 0.98 & $2.7005(17)$ & 1.78 & $155^{\prime}$ & - \\
\hline & $\mathrm{O}(5)-\mathrm{H}(5 \mathrm{O}) \cdots \mathrm{N}(4)$ & 0.98 & $2.7533(18)$ & 1.77 & 175 & $-1-x, 2-y,-z$ \\
\hline
\end{tabular}




\begin{tabular}{|c|c|c|c|c|c|c|}
\hline & $\mathrm{O}(6)-\mathrm{H}(6 \mathrm{O}) \cdots \mathrm{O}(8)$ & 0.98 & $2.6567(17)$ & 1.68 & 173 & - \\
\hline & $\mathrm{O}(8)-\mathrm{H}(8 \mathrm{~A}) \cdots \mathrm{N}(6)$ & 0.98 & $2.832(2)$ & 1.89 & 160 & $1+\mathrm{x}, \mathrm{y}, \mathrm{z}$ \\
\hline & $\mathrm{O}(8)-\mathrm{H}(8 \mathrm{~B}) \cdots \mathrm{N}(1)$ & 0.98 & $2.896(2)$ & 1.95 & 160 & - \\
\hline & $\mathrm{C}(40)-\mathrm{H}(40) \cdots \mathrm{O}(3)$ & 1.08 & $3.271(2)$ & 2.42 & 134 & $1+\mathrm{x},-1+\mathrm{y}, \mathrm{z}$ \\
\hline \multirow{7}{*}{$\begin{array}{c}\text { QUE:DPE-I } \\
\text { Form I }\end{array}$} & $\mathrm{O}(1)-\mathrm{H}(1 \mathrm{O}) \cdots \mathrm{N}(1)$ & 0.98 & $2.655(3)$ & 1.67 & 175 & $-\mathrm{x}, 1-\mathrm{y},-\mathrm{z}$ \\
\hline & $\mathrm{O}(4)-\mathrm{H}(4 \mathrm{O}) \cdots \mathrm{O}(3)$ & 0.98 & $2.759(2)$ & 1.89 & $146^{\prime}$ & $1-\mathrm{x},-\mathrm{y},-\mathrm{z}$ \\
\hline & $\mathrm{O}(5)-\mathrm{H}(5 \mathrm{O}) \cdots \mathrm{O}(1)$ & 0.98 & $2.7624(19)$ & 1.84 & 156 & $\mathrm{x}, \mathrm{y}, 1+\mathrm{z}$ \\
\hline & $\mathrm{O}(6)-\mathrm{H}(6 \mathrm{O}) \cdots \mathrm{N}(2)$ & 0.98 & $2.694(3)$ & 1.71 & 175 & - \\
\hline & $\mathrm{C}(14)-\mathrm{H}(14) \cdots \mathrm{O}(8)$ & 1.08 & $3.416(3)$ & 2.48 & 144 & $-1+\mathrm{x}, \mathrm{y}, \mathrm{z}$ \\
\hline & $\mathrm{C}(26)-\mathrm{H}(26) \cdots \mathrm{O}(2)$ & 1.08 & $3.350(2)$ & 2.40 & 145 & $-\mathrm{x}, 1-\mathrm{y},-\mathrm{z}$ \\
\hline & $\mathrm{C}(31)-\mathrm{H}(31 \mathrm{~A}) \cdots \mathrm{O}(7)$ & 1.08 & $3.474(3)$ & 2.51 & 147 & $\mathrm{x}, 1+\mathrm{y}, 1+\mathrm{z}$ \\
\hline \multirow{6}{*}{$\begin{array}{c}\text { QUE:DPE-I } \\
\text { Form II }\end{array}$} & $\mathrm{O}(1)-\mathrm{H}(1 \mathrm{O}) \cdots \mathrm{N}(2)$ & 0.98 & $2.657(3)$ & 1.68 & 173 & - \\
\hline & $\mathrm{O}(4)-\mathrm{H}(4 \mathrm{O}) \cdots \mathrm{O}(3)$ & 0.98 & $2.752(2)$ & 1.91 & $142^{\prime}$ & $-x, 1-y, 1-z$ \\
\hline & $\mathrm{O}(5)-\mathrm{H}(5 \mathrm{O}) \cdots \mathrm{O}(1)$ & 0.98 & $2.746(2)$ & 1.85 & 150 & $\mathrm{x}, \mathrm{y}, 1+\mathrm{z}$ \\
\hline & $\mathrm{O}(6)-\mathrm{H}(6 \mathrm{O}) \cdots \mathrm{N}(1)$ & 0.98 & $2.704(3)$ & 1.73 & 171 & $3-x,-y, 1-z$ \\
\hline & $\mathrm{C}(14)-\mathrm{H}(14) \cdots \mathrm{O}(8)$ & 1.08 & $3.383(4)$ & 2.48 & 140 & $2-x, 1-y, 1-z$ \\
\hline & $\mathrm{C}(20)-\mathrm{H}(20) \cdots \mathrm{O}(2)$ & 1.08 & $3.375(3)$ & 2.42 & 146 & $1-x, 1-y,-z$ \\
\hline \multirow{6}{*}{$\begin{array}{c}\text { QUE:DPE-I } \\
\text { Form III }\end{array}$} & $\mathrm{O}(1)-\mathrm{H}(1 \mathrm{O}) \cdots \mathrm{N}(1)$ & 0.98 & $2.642(3)$ & 1.66 & 176 & - \\
\hline & $\mathrm{O}(4)-\mathrm{H}(4 \mathrm{O}) \cdots \mathrm{O}(3)$ & 0.98 & $2.758(3)$ & 1.91 & $143^{\prime}$ & $2-x,-y,-z$ \\
\hline & $\mathrm{O}(5)-\mathrm{H}(5 \mathrm{O}) \cdots \mathrm{O}(1)$ & 0.98 & $2.769(3)$ & 1.88 & 149 & $\mathrm{x}, \mathrm{y},-1+\mathrm{z}$ \\
\hline & $\mathrm{O}(6)-\mathrm{H}(6 \mathrm{O}) \cdots \mathrm{N}(2)$ & 0.98 & $2.693(3)$ & 1.71 & 178 & $-1-x, 1-y,-z$ \\
\hline & $\mathrm{C}(16)-\mathrm{H}(16) \cdots \mathrm{O}(8)$ & 1.08 & $3.203(5)$ & 2.20 & 154 & $1-x, 1-y,-z$ \\
\hline & $\mathrm{C}(20)-\mathrm{H}(20) \cdots \mathrm{O}(2)$ & 1.08 & $3.325(3)$ & 2.35 & 149 & $1-x,-y, 1-z$ \\
\hline \multirow{6}{*}{$\begin{array}{c}\text { QUE:DPE-I } \\
\text { Form IV }\end{array}$} & $\mathrm{O}(1)-\mathrm{H}(1 \mathrm{O}) \cdots \mathrm{N}(2)$ & 0.98 & $2.7238(19)$ & 1.75 & 171 & $-\mathrm{x}, 1-\mathrm{y}, 1-\mathrm{z}$ \\
\hline & $\mathrm{O}(4)-\mathrm{H}(4 \mathrm{O}) \cdots \mathrm{N}(4)$ & 0.98 & $2.6921(19)$ & 1.74 & $161^{\prime}$ & $1-\mathrm{x}, 1-\mathrm{y},-\mathrm{z}$ \\
\hline & $\mathrm{O}(5)-\mathrm{H}(5 \mathrm{O}) \cdots \mathrm{N}(3)$ & 0.98 & $2.7283(19)$ & 1.75 & 172 & $\mathrm{x}, 1+\mathrm{y}, \mathrm{z}$ \\
\hline & $\mathrm{O}(6)-\mathrm{H}(6 \mathrm{O}) \cdots \mathrm{N}(1)$ & 0.98 & $2.759(2)$ & 1.78 & 173 & $x, 3 / 2-y, 1 / 2+z$ \\
\hline & $\mathrm{C}(28)-\mathrm{H}(28) \cdots \mathrm{O}(5)$ & 1.08 & $3.3389(19)$ & 2.41 & 143 & 1-x,1-y,1-z \\
\hline & $\mathrm{C}(37)-\mathrm{H}(37) \cdots \mathrm{O}(3)$ & 1.08 & $3.014(2)$ & 2.34 & 119 & $1-x, 1-y,-z$ \\
\hline \multirow{6}{*}{$\begin{array}{c}\text { QUE:DPE-I } \\
\text { Form V }\end{array}$} & $\mathrm{O}(1)-\mathrm{H}(1 \mathrm{O}) \cdots \mathrm{N}(1)$ & 0.98 & $2.763(3)$ & 1.78 & 173 & $1 / 2-\mathrm{x}, 1 / 2+\mathrm{y}, 1 / 2-\mathrm{z}$ \\
\hline & $\mathrm{O}(4)-\mathrm{H}(4 \mathrm{O}) \cdots \mathrm{O}(5)$ & 0.98 & $2.690(2)$ & 1.79 & $151^{\prime}$ & $-\mathrm{x}, \mathrm{y},-1 / 2-\mathrm{z}$ \\
\hline & $\mathrm{O}(5)-\mathrm{H}(5 \mathrm{O}) \cdots \mathrm{O}(2)$ & 0.98 & $2.797(2)$ & 1.94 & $144^{\prime}$ & $-1 / 2+x, 3 / 2-y,-1 / 2+z$ \\
\hline & $\mathrm{O}(6)-\mathrm{H}(6 \mathrm{O}) \cdots \mathrm{N}(2)$ & 0.98 & $2.663(3)$ & 1.71 & 162 & $-1 / 2-x, 1 / 2-y,-z$ \\
\hline & $\mathrm{C}(4)-\mathrm{H}(4) \cdots \mathrm{N}(1)$ & 1.08 & $3.287(3)$ & 2.50 & 128 & $1 / 2-\mathrm{x}, 1 / 2+\mathrm{y}, 1 / 2-\mathrm{z}$ \\
\hline & $\mathrm{C}(20)-\mathrm{H}(20) \cdots \mathrm{O}(3)$ & 1.08 & $3.294(3)$ & 2.50 & 130 & $1 / 2-x, 1 / 2-y,-z$ \\
\hline \multirow{5}{*}{$\begin{array}{c}\text { QUE:DPE-II } \\
\text { Form I }\end{array}$} & $\mathrm{O}(1)-\mathrm{H}(1 \mathrm{O}) \cdots \mathrm{N}(2)$ & 0.98 & $2.657(2)$ & 1.68 & 172 & $-x, 1-y, 2-z$ \\
\hline & $\mathrm{O}(4)-\mathrm{H}(4 \mathrm{O}) \cdots \mathrm{O}(3)$ & 0.98 & $2.722(2)$ & 1.88 & $141^{\prime}$ & $2-x,-y, 2-z$ \\
\hline & $\mathrm{O}(6)-\mathrm{H}(5 \mathrm{O}) \cdots \mathrm{O}(1)$ & 0.98 & $2.7887(19)$ & 1.89 & 151 & $\mathrm{x}, \mathrm{y},-1+\mathrm{z}$ \\
\hline & $\mathrm{O}(7)-\mathrm{H}(6 \mathrm{O}) \cdots \mathrm{N}(1)$ & 0.98 & $2.702(2)$ & 1.72 & 178 & $-1+\mathrm{x}, \mathrm{y}, \mathrm{z}$ \\
\hline & $\mathrm{C}(17)-\mathrm{H}(17) \cdots \mathrm{O}(1)$ & 1.08 & $3.320(3)$ & 2.47 & 134 & $1-x, 1-y, 2-z$ \\
\hline
\end{tabular}




\begin{tabular}{|c|c|c|c|c|c|c|}
\hline & $\mathrm{C}(25)-\mathrm{H}(25) \cdots \mathrm{O}(2)$ & 1.08 & $3.426(2)$ & 2.49 & 144 & $-1+\mathrm{x}, 1+\mathrm{y},-1+\mathrm{z}$ \\
\hline \multirow{8}{*}{$\begin{array}{c}\text { QUE:DPE-II } \\
\text { Form II }\end{array}$} & $\mathrm{O}(1)-\mathrm{H}(1 \mathrm{O}) \cdots \mathrm{N}(2)$ & 0.98 & $2.6962(18)$ & 1.72 & 174 & $1-\mathrm{x}, 1-\mathrm{y},-\mathrm{z}$ \\
\hline & $\mathrm{O}(4)-\mathrm{H}(4 \mathrm{O}) \cdots \mathrm{N}(3)$ & 0.98 & $2.6367(18)$ & 1.68 & $163^{\prime}$ & - \\
\hline & $\mathrm{O}(5)-\mathrm{H}(5 \mathrm{O}) \cdots \mathrm{N}(4)$ & 0.98 & $2.7009(18)$ & 1.73 & 170 & $-x, 1-y, 1-z$ \\
\hline & $\mathrm{O}(6)-\mathrm{H}(6 \mathrm{O}) \cdots \mathrm{N}(1)$ & 0.98 & $2.7236(18)$ & 1.75 & 171 & $\mathrm{x}, 1 / 2-\mathrm{y},-1 / 2+\mathrm{z}$ \\
\hline & $\mathrm{C}(4)-\mathrm{H}(4) \cdots \mathrm{O}(2)$ & 1.08 & $3.4732(19)$ & 2.43 & 160 & $1-x, 2-y, 1-z$ \\
\hline & $\mathrm{C}(28)-\mathrm{H}(28) \cdots \mathrm{O}(6)$ & 1.08 & $3.295(2)$ & 2.43 & 136 & $\mathrm{x}, 1 / 2-\mathrm{y}, 1 / 2+\mathrm{z}$ \\
\hline & $\mathrm{C}(37)-\mathrm{H}(37) \cdots \mathrm{O}(5)$ & 1.08 & $3.386(2)$ & 2.30 & 177 & $\mathrm{x}, \mathrm{y}, 1+\mathrm{z}$ \\
\hline & $\mathrm{C}(39)-\mathrm{H}(39) \cdots \mathrm{O}(6)$ & 1.08 & $3.253(2)$ & 2.41 & 134 & $-\mathrm{x}, 1 / 2+\mathrm{y}, 1 / 2-\mathrm{z}$ \\
\hline \multirow{14}{*}{$\begin{array}{c}\text { QUE:DPE-II } \\
\text { Form III }\end{array}$} & $\mathrm{O}(1)-\mathrm{H}(1 \mathrm{O}) \cdots \mathrm{N}(3)$ & 0.98 & $2.666(3)$ & 1.70 & 166 & $1-\mathrm{x},-1 / 2+\mathrm{y}, 5 / 2-\mathrm{z}$ \\
\hline & $\mathrm{O}(4)-\mathrm{H}(4 \mathrm{O}) \cdots \mathrm{O}(10)$ & 0.98 & $2.7504(19)$ & 1.90 & $143^{\prime}$ & - \\
\hline & $\mathrm{O}(5)-\mathrm{H}(5 \mathrm{O}) \cdots \mathrm{N}(4)$ & 0.98 & $2.742(3)$ & 1.76 & 173 & $-\mathrm{x}, 1-\mathrm{y}, 2-\mathrm{z}$ \\
\hline & $\mathrm{O}(6)-\mathrm{H}(6 \mathrm{O}) \cdots \mathrm{O}(4)$ & 0.98 & $2.883(2)$ & 2.14 & $131^{\prime}$ & $-\mathrm{x}, 1 / 2+\mathrm{y}, 5 / 2-\mathrm{z}$ \\
\hline & $\mathrm{O}(8)-\mathrm{H}(8 \mathrm{O}) \cdots \mathrm{N}(1)$ & 0.98 & $2.619(2)$ & 1.65 & 167 & $-\mathrm{x},-\mathrm{y}, 2-\mathrm{z}$ \\
\hline & $\mathrm{O}(9)-\mathrm{H}(9 \mathrm{O}) \cdots \mathrm{O}(6)$ & 0.98 & $3.033(2)$ & 2.41 & $120^{\prime}$ & $-\mathrm{x},-1 / 2+\mathrm{y}, 5 / 2-\mathrm{z}$ \\
\hline & $\mathrm{O}(11)-\mathrm{H}(11 \mathrm{O}) \cdots \mathrm{O}(3)$ & 0.98 & $2.7228(19)$ & 1.82 & 150 & - \\
\hline & $\mathrm{O}(12)-\mathrm{H}(12 \mathrm{O}) \cdots \mathrm{N}(2)$ & 0.98 & $2.699(2)$ & 1.72 & 170 & $1-\mathrm{x}, 1 / 2+\mathrm{y}, 5 / 2-\mathrm{z}$ \\
\hline & $\mathrm{O}(13)-\mathrm{H}(13 \mathrm{O}) \cdots \mathrm{O}(8)$ & 0.98 & $2.9042(19)$ & 2.09 & 139 & $1+\mathrm{x}, \mathrm{y}, \mathrm{z}$ \\
\hline & $\mathrm{C}(4)-\mathrm{H}(4) \cdots \mathrm{O}(7)$ & 1.08 & $3.380(2)$ & 2.43 & 146 & $1-x,-1 / 2+y, 5 / 2-z$ \\
\hline & $\mathrm{C}(36)-\mathrm{H}(36 \mathrm{~B}) \cdots \mathrm{O}(14)$ & 1.08 & $3.461(3)$ & 2.45 & 154 & - \\
\hline & $\mathrm{C}(47)-\mathrm{H}(47) \cdots \mathrm{O}(13)$ & 1.08 & $3.397(3)$ & 2.36 & 159 & $1-x,-y, 2-z$ \\
\hline & $\mathrm{C}(50)-\mathrm{H}(50) \cdots \mathrm{O}(11)$ & 1.08 & $3.460(3)$ & 2.43 & 158 & $1-x,-1 / 2+y, 5 / 2-z$ \\
\hline & $\mathrm{C}(54)-\mathrm{H}(54) \cdots \mathrm{O}(9)$ & 1.08 & $3.232(3)$ & 2.32 & 141 & $-\mathrm{x},-1 / 2+\mathrm{y}, 5 / 2-\mathrm{z}$ \\
\hline \multirow{6}{*}{$\begin{array}{c}\text { QUE:44AP } \\
\text { Form I }\end{array}$} & $\mathrm{O}(1)-\mathrm{H}(1 \mathrm{O}) \cdots \mathrm{N}(4)$ & 0.98 & $2.685(6)$ & 1.70 & 175 & $1-\mathrm{x}, 1-\mathrm{y},-\mathrm{z}$ \\
\hline & $\mathrm{O}(4)-\mathrm{H}(4 \mathrm{O}) \cdots \mathrm{O}(3)$ & 0.98 & $2.729(5)$ & 1.80 & $157^{\prime}$ & $2-\mathrm{x},-\mathrm{y},-\mathrm{z}$ \\
\hline & $\mathrm{O}(5)-\mathrm{H}(5 \mathrm{O}) \cdots \mathrm{O}(1)$ & 0.98 & $2.756(4)$ & 1.85 & 151 & $\mathrm{x}, \mathrm{y}, 1+\mathrm{z}$ \\
\hline & $\mathrm{O}(6)-\mathrm{H}(6 \mathrm{O}) \cdots \mathrm{N}(1)$ & 0.98 & $2.719(6)$ & 1.74 & 175 & $-1+\mathrm{x}, \mathrm{y}, \mathrm{z}$ \\
\hline & $\mathrm{C}(14)-\mathrm{H}(14) \cdots \mathrm{O}(8)$ & 1.08 & $3.267(7)$ & 2.39 & 137 & $1-x, 1-y,-z$ \\
\hline & $\mathrm{C}(21)-\mathrm{H}(21) \cdots \mathrm{O}(2)$ & 1.08 & $3.404(5)$ & 2.44 & 148 & $\mathrm{x}, 1+\mathrm{y}, 1+\mathrm{z}$ \\
\hline \multirow{6}{*}{$\begin{array}{c}\text { QUE:44AP } \\
\text { Form II }\end{array}$} & $\mathrm{O}(1)-\mathrm{H}(1 \mathrm{O}) \cdots \mathrm{N}(4)$ & 0.98 & $2.791(3)$ & 1.81 & 174 & - \\
\hline & $\mathrm{O}(4)-\mathrm{H}(4 \mathrm{O}) \cdots \mathrm{O}(5)$ & 0.98 & $2.669(2)$ & 1.79 & $148^{\prime}$ & $-\mathrm{x}, \mathrm{y},-1 / 2-\mathrm{z}$ \\
\hline & $\mathrm{O}(5)-\mathrm{H}(5 \mathrm{O}) \cdots \mathrm{O}(2)$ & 0.98 & $2.759(2)$ & 1.92 & $141^{\prime}$ & $-1 / 2+x, 1 / 2-y,-1 / 2+z$ \\
\hline & $\mathrm{O}(6)-\mathrm{H}(6 \mathrm{O}) \cdots \mathrm{N}(1)$ & 0.98 & $2.690(3)$ & 1.76 & 156 & $-1+x, 1-y,-1 / 2+z$ \\
\hline & $\mathrm{C}(4)-\mathrm{H}(4) \cdots \mathrm{N}(4)$ & 1.08 & $3.292(3)$ & 2.50 & 124 & - \\
\hline & $\mathrm{C}(24)-\mathrm{H}(24) \cdots \mathrm{O}(3)$ & 1.08 & $3.294(3)$ & 2.51 & 128 & $\mathrm{x}, 1-\mathrm{y}, 1 / 2+\mathrm{z}$ \\
\hline \multirow{5}{*}{$\begin{array}{c}\text { QUE:PHE } \\
\text { Form I }\end{array}$} & $\mathrm{O}(1)-\mathrm{H}(1 \mathrm{O}) \cdots \mathrm{N}(2)$ & 0.98 & $2.760(3)$ & 1.78 & 173 & $-\mathrm{x}, 1-\mathrm{y}, 1-\mathrm{z}$ \\
\hline & $\mathrm{O}(4)-\mathrm{H}(4 \mathrm{O}) \cdots \mathrm{O}(8)$ & 0.98 & $2.704(2)$ & 1.91 & $136^{\prime}$ & - \\
\hline & $\mathrm{O}(5)-\mathrm{H}(5) \cdots \mathrm{O}(1)$ & 0.98 & $2.765(2)$ & 1.85 & 153 & $\mathrm{x}, \mathrm{y},-1+\mathrm{z}$ \\
\hline & $\mathrm{O}(6)-\mathrm{H}(6 \mathrm{O}) \cdots \mathrm{N}(1)$ & 0.98 & $2.796(3)$ & 1.83 & 167 & $-1+\mathrm{x}, \mathrm{y}, \mathrm{z}$ \\
\hline & $\mathrm{C}(17)-\mathrm{H}(17) \cdots \mathrm{O}(2)$ & 1.08 & $3.313(3)$ & 2.46 & 135 & $1-x,-y, 1-z$ \\
\hline
\end{tabular}




\begin{tabular}{|c|c|c|c|c|c|c|}
\hline & $\mathrm{C}(24)-\mathrm{H}(24) \cdots \mathrm{O}(5)$ & 1.08 & $3.362(3)$ & 2.45 & 141 & $1-x, 1-y,-z$ \\
\hline \multirow{4}{*}{$\begin{array}{c}\text { QUE:PHE } \\
\text { Form II }\end{array}$} & $\mathrm{O}(1)-\mathrm{H}(1 \mathrm{O}) \cdots \mathrm{N}(1)$ & 0.98 & $2.722(5)$ & 1.74 & 177 & $1-x, 1-y, 1-z$ \\
\hline & $\mathrm{O}(5)-\mathrm{H}(5 \mathrm{O}) \cdots \mathrm{O}(1)$ & 0.98 & $2.737(4)$ & 1.80 & 159 & $\mathrm{x}, \mathrm{y}, 1+\mathrm{z}$ \\
\hline & $\mathrm{O}(6)-\mathrm{H}(6 \mathrm{~A}) \cdots \mathrm{N}(2)$ & 0.98 & $2.786(5)$ & 1.82 & 165 & $1+\mathrm{x},-1+\mathrm{y}, 1+\mathrm{z}$ \\
\hline & $\mathrm{C}(20)-\mathrm{H}(20) \cdots \mathrm{O}(2)$ & 1.08 & $3.263(6)$ & 2.46 & 130 & $-\mathrm{x}, 2-\mathrm{y}, 1-\mathrm{z}$ \\
\hline \multirow{7}{*}{$\begin{array}{c}\text { QUE:PHE } \\
\text { Form III }\end{array}$} & $\mathrm{O}(1)-\mathrm{H}(1 \mathrm{O}) \cdots \mathrm{N}(4)$ & 0.98 & $2.7694(18)$ & 1.81 & 164 & 1-x,1-y,1-z \\
\hline & $\mathrm{O}(4)-\mathrm{H}(4 \mathrm{O}) \cdots \mathrm{N}(5)$ & 0.98 & $2.6997(17)$ & 1.80 & $150^{\prime}$ & - \\
\hline & $\mathrm{O}(5)-\mathrm{H}(5 \mathrm{O}) \cdots \mathrm{N}(7)$ & 0.98 & $2.9068(17)$ & 1.93 & 174 & - \\
\hline & $\mathrm{O}(6)-\mathrm{H}(6 \mathrm{O}) \cdots \mathrm{O}(8)$ & 0.98 & $2.6122(18)$ & 1.67 & $159^{\prime}$ & - \\
\hline & $\mathrm{O}(8)-\mathrm{H}(8 \mathrm{O}) \cdots \mathrm{N}(6)$ & 0.98 & $2.811(2)$ & 1.83 & 177 & $-\mathrm{x}, 2-\mathrm{y},-\mathrm{z}$ \\
\hline & $\mathrm{C}(14)-\mathrm{H}(14) \cdots \mathrm{N}(1)$ & 1.08 & $3.464(2)$ & 2.41 & 164 & - \\
\hline & $\mathrm{C}(20)-\mathrm{H}(20) \cdots \mathrm{O}(3)$ & 1.08 & $3.3386(19)$ & 2.34 & 152 & $1+x,-1+y, z$ \\
\hline \multirow{6}{*}{$\begin{array}{c}\text { QUE:PHE } \\
\text { Form IV }\end{array}$} & $\mathrm{O}(1)-\mathrm{H}(1 \mathrm{O}) \cdots \mathrm{N}(5)$ & 0.98 & $2.736(2)$ & 1.76 & 169 & - \\
\hline & $\mathrm{O}(4)-\mathrm{H}(4 \mathrm{O}) \cdots \mathrm{N}(11)$ & 0.98 & $2.850(2)$ & 1.95 & $152^{\prime}$ & $3-x, 1-y, 1-z$ \\
\hline & $\mathrm{O}(5)-\mathrm{H}(5 \mathrm{O}) \cdots \mathrm{N}(12)$ & 0.98 & $2.861(2)$ & 1.89 & 171 & $\mathrm{x}, 1+\mathrm{y}, 1+\mathrm{z}$ \\
\hline & $\mathrm{O}(6)-\mathrm{H}(6 \mathrm{O}) \cdots \mathrm{O}(8)$ & 0.98 & $2.715(2)$ & 1.74 & 174 & - \\
\hline & $\mathrm{C}(19)-\mathrm{H}(19) \cdots \mathrm{O}(6)$ & 1.08 & $3.347(3)$ & 2.45 & 139 & $1+x,-1+y,-1+z$ \\
\hline & $\mathrm{C}(28)-\mathrm{H}(28) \cdots \mathrm{O}(3)$ & 1.08 & $3.134(2)$ & 2.16 & 149 & $-1+\mathrm{x}, \mathrm{y},-1+\mathrm{z}$ \\
\hline \multirow{6}{*}{$\begin{array}{c}\text { QUE:PHE } \\
\text { Form V }\end{array}$} & $\mathrm{O}(1)-\mathrm{H}(1 \mathrm{O}) \cdots \mathrm{N}(2)$ & 0.98 & $2.792(3)$ & 1.85 & 159 & $-\mathrm{x},-\mathrm{y}, 1-\mathrm{z}$ \\
\hline & $\mathrm{O}(4)-\mathrm{H}(4 \mathrm{O}) \cdots \mathrm{O}(3)$ & 0.98 & $2.699(3)$ & 1.82 & $147^{\prime}$ & $2-x, 1-y, 1-z$ \\
\hline & $\mathrm{O}(5)-\mathrm{H}(5 \mathrm{O}) \cdots \mathrm{O}(6)$ & 0.98 & $2.792(3)$ & 1.98 & $139^{\prime}$ & $-\mathrm{x},-\mathrm{y},-\mathrm{z}$ \\
\hline & $\mathrm{O}(6)-\mathrm{H}(6 \mathrm{O}) \cdots \mathrm{N}(1)$ & 0.98 & $2.743(3)$ & 1.78 & 167 & - \\
\hline & $\mathrm{C}(18)-\mathrm{H}(18) \cdots \mathrm{O}(7)$ & 1.08 & $3.458(3)$ & 2.44 & 156 & $-1+\mathrm{x}, \mathrm{y}, \mathrm{z}$ \\
\hline & $\mathrm{C}(24)-\mathrm{H}(24) \cdots \mathrm{N}(3)$ & 1.08 & $3.353(4)$ & 2.33 & 157 & $\mathrm{x},-1+\mathrm{y}, \mathrm{z}$ \\
\hline \multirow{5}{*}{ QUE:44BP:22TP } & $\mathrm{O}(1)-\mathrm{H}(1 \mathrm{O}) \cdots \mathrm{N}(2)$ & 0.98 & $2.658(3)$ & 1.68 & 176 & $2-x, 1-y,-z$ \\
\hline & $\mathrm{O}(4)-\mathrm{H}(4 \mathrm{O}) \cdots \mathrm{O}(3)$ & 0.98 & $2.667(3)$ & 1.80 & $145^{\prime}$ & $-\mathrm{x}, 2-\mathrm{y},-\mathrm{z}$ \\
\hline & $\mathrm{O}(5)-\mathrm{H}(5 \mathrm{O}) \cdots \mathrm{O}(1)$ & 0.98 & $2.737(2)$ & 1.80 & 158 & $\mathrm{x}, \mathrm{y},-1+\mathrm{z}$ \\
\hline & $\mathrm{O}(6)-\mathrm{H}(6 \mathrm{O}) \cdots \mathrm{N}(1)$ & 0.98 & $2.706(3)$ & 1.73 & 171 & - \\
\hline & $\mathrm{C}(17)-\mathrm{H}(17) \cdots \mathrm{O}(5)$ & 1.08 & $3.275(2)$ & 2.33 & 144 & $1-x, 1-y,-1-z$ \\
\hline \multirow{11}{*}{ QUE:44BP:TTF } & $\mathrm{O}(1)-\mathrm{H}(1 \mathrm{O}) \cdots \mathrm{N}(1)$ & 0.98 & $2.672(4)$ & 1.71 & 165 & $1-\mathrm{x},-\mathrm{y}, 1-\mathrm{z}$ \\
\hline & $\mathrm{O}(4)-\mathrm{H}(4 \mathrm{O}) \cdots \mathrm{N}(5)$ & 0.98 & $2.679(4)$ & 1.73 & $161^{\prime}$ & - \\
\hline & $\mathrm{O}(5)-\mathrm{H}(5 \mathrm{O}) \cdots \mathrm{N}(4)$ & 0.98 & $2.733(5)$ & 1.75 & 175 & $-1+\mathrm{x}, \mathrm{y}, 1+\mathrm{z}$ \\
\hline & $\mathrm{O}(6)-\mathrm{H}(6 \mathrm{O}) \cdots \mathrm{O}(1)$ & 0.98 & $2.794(3)$ & 1.99 & 137 & $\mathrm{x}, 1+\mathrm{y}, \mathrm{z}$ \\
\hline & $\mathrm{O}(8)-\mathrm{H}(8 \mathrm{O}) \cdots \mathrm{N}(2)$ & 0.98 & $2.693(4)$ & 1.75 & 159 & $-x, 1-y, 1-z$ \\
\hline & $\mathrm{O}(11)-\mathrm{H}(11 \mathrm{O}) \cdots \mathrm{N}(3)$ & 0.98 & $2.841(4)$ & 1.91 & $157^{\prime}$ & - \\
\hline & $\mathrm{O}(12)-\mathrm{H}(12 \mathrm{O}) \cdots \mathrm{N}(6)$ & 0.98 & $2.703(4)$ & 1.72 & 175 & $1+\mathrm{x}, \mathrm{y},-1+\mathrm{z}$ \\
\hline & $\mathrm{O}(13)-\mathrm{H}(13 \mathrm{O}) \cdots \mathrm{O}(8)$ & 0.98 & $2.807(3)$ & 1.99 & 139 & $\mathrm{x},-1+\mathrm{y}, \mathrm{z}$ \\
\hline & $\mathrm{C}(4)-\mathrm{H}(4) \cdots \mathrm{O}(5)$ & 1.08 & $3.342(5)$ & 2.35 & 152 & $\mathrm{x},-1+\mathrm{y}, \mathrm{z}$ \\
\hline & $\mathrm{C}(14)-\mathrm{H}(14) \cdots \mathrm{S}(2)$ & 1.08 & $3.557(3)$ & 2.64 & 142 & - \\
\hline & $\mathrm{C}(15)-\mathrm{H}(15) \cdots \mathrm{S}(3)$ & 1.08 & $3.439(3)$ & 2.66 & 128 & $1-x,-y, 1-z$ \\
\hline
\end{tabular}




\begin{tabular}{|c|c|c|c|c|c|c|}
\hline & $\mathrm{C}(19)-\mathrm{H}(19) \cdots \mathrm{O}(12)$ & 1.08 & $3.367(3)$ & 2.36 & 155 & $\mathrm{x}, 1+\mathrm{y}, \mathrm{z}$ \\
\hline & $\mathrm{C}(41)-\mathrm{H}(41) \cdots \mathrm{O}(10)$ & 1.08 & $3.102(4)$ & 2.40 & 121 & - \\
\hline & $\mathrm{C}(41)-\mathrm{H}(41) \cdots \mathrm{S}(6)$ & 1.08 & $3.625(4)$ & 2.77 & $136^{\prime}$ & $1-x, 1-y,-z$ \\
\hline & $\mathrm{C}(48)-\mathrm{H}(48) \cdots \mathrm{O}(2)$ & 1.08 & $3.051(5)$ & 2.46 & 113 & $1+\mathrm{x}, 1+\mathrm{y},-1+\mathrm{z}$ \\
\hline \multirow{5}{*}{ QUE:DPE-I:22TP } & $\mathrm{O}(1)-\mathrm{H}(1 \mathrm{O}) \cdots \mathrm{N}(1)$ & 0.98 & $2.659(3)$ & 1.68 & 175 & - \\
\hline & $\mathrm{O}(4)-\mathrm{H}(4 \mathrm{O}) \cdots \mathrm{O}(3)$ & 0.98 & $2.730(3)$ & 1.84 & $148^{\prime}$ & $-\mathrm{x}, 1-\mathrm{y}, 1-\mathrm{z}$ \\
\hline & $\mathrm{O}(5)-\mathrm{H}(5 \mathrm{O}) \cdots \mathrm{O}(1)$ & 0.98 & $2.759(3)$ & 1.84 & 154 & $\mathrm{x}, \mathrm{y}, 1+\mathrm{z}$ \\
\hline & $\mathrm{O}(6)-\mathrm{H}(6 \mathrm{O}) \cdots \mathrm{N}(2)$ & 0.98 & $2.706(4)$ & 1.73 & 169 & $3-x,-y, 1-z$ \\
\hline & $\mathrm{C}(20)-\mathrm{H}(20) \cdots \mathrm{O}(2)$ & 1.08 & $3.344(3)$ & 2.36 & 150 & $1-x, 1-y,-z$ \\
\hline \multirow{17}{*}{ QUE:DPE-I:PYR } & $\mathrm{O}(1)-\mathrm{H}(1 \mathrm{O}) \cdots \mathrm{N}(1)$ & 0.98 & $2.640(4)$ & 1.68 & 166 & - \\
\hline & $\mathrm{O}(4)-\mathrm{H}(4 \mathrm{O}) \cdots \mathrm{N}(6)$ & 0.98 & $2.750(4)$ & 1.79 & $166^{\prime}$ & $2-x, 1-y,-z$ \\
\hline & $\mathrm{O}(5)-\mathrm{H}(5 \mathrm{O}) \cdots \mathrm{N}(4)$ & 0.98 & $2.759(4)$ & 1.79 & 169 & $2-x,-y,-z$ \\
\hline & $\mathrm{O}(6)-\mathrm{H}(6 \mathrm{O}) \cdots \mathrm{O}(1)$ & 0.98 & $2.766(4)$ & 1.95 & 139 & $\mathrm{x},-1+\mathrm{y}, \mathrm{z}$ \\
\hline & $\mathrm{O}(8)-\mathrm{H}(8 \mathrm{O}) \cdots \mathrm{N}(2)$ & 0.98 & $2.653(4)$ & 1.69 & 167 & $-\mathrm{x}, 1-\mathrm{y}, 1-\mathrm{z}$ \\
\hline & $\mathrm{O}(11)-\mathrm{H}(11 \mathrm{O}) \cdots \mathrm{N}(3)$ & 0.98 & $2.830(5)$ & 1.99 & $141^{\prime}$ & - \\
\hline & $\mathrm{O}(12)-\mathrm{H}(12 \mathrm{O}) \cdots \mathrm{N}(5)$ & 0.98 & $2.732(4)$ & 1.76 & 170 & $\mathrm{x},-1+\mathrm{y}, \mathrm{z}$ \\
\hline & $\mathrm{O}(13)-\mathrm{H}(13 \mathrm{O}) \cdots \mathrm{O}(8)$ & 0.98 & $2.772(4)$ & 1.97 & 137 & $\mathrm{x},-1+\mathrm{y}, \mathrm{z}$ \\
\hline & $\mathrm{C}(4)-\mathrm{H}(4) \cdots \mathrm{O}(5)$ & 1.08 & $3.450(5)$ & 2.46 & 151 & $\mathrm{x}, 1+\mathrm{y}, \mathrm{z}$ \\
\hline & $\mathrm{C}(35)-\mathrm{H}(35) \cdots \mathrm{O}(6)$ & 1.08 & $3.449(5)$ & 2.47 & 150 & $\mathrm{x}, 1+\mathrm{y}, \mathrm{z}$ \\
\hline & $\mathrm{C}(41)-\mathrm{H}(41) \cdots \mathrm{O}(13)$ & 1.08 & $3.391(5)$ & 2.41 & 149 & $-\mathrm{x},-\mathrm{y}, 1-\mathrm{z}$ \\
\hline & $\mathrm{C}(47)-\mathrm{H}(47) \cdots \mathrm{O}(10)$ & 1.08 & $3.093(5)$ & 2.32 & 127 & - \\
\hline & $\mathrm{C}(53)-\mathrm{H}(53) \cdots \mathrm{O}(2)$ & 1.08 & $3.299(5)$ & 2.35 & 145 & $2-x, 1-y,-z$ \\
\hline & $\mathrm{C}(55)-\mathrm{H}(55) \cdots \mathrm{O}(9)$ & 1.08 & $3.328(5)$ & 2.35 & 150 & - \\
\hline & $\mathrm{C}(73)-\mathrm{H}(73) \cdots \mathrm{O}(3)$ & 1.08 & $3.313(5)$ & 2.46 & 135 & $-1+\mathrm{x}, \mathrm{y}, \mathrm{z}$ \\
\hline & $\mathrm{C}(75)-\mathrm{H}(75) \cdots \mathrm{O}(1)$ & 1.08 & $3.506(5)$ & 2.44 & 168 & - \\
\hline & $\mathrm{C}(83)-\mathrm{H}(83) \cdots \mathrm{O}(12)$ & 1.08 & $3.404(5)$ & 2.46 & 144 & $\mathrm{x}, 1+\mathrm{y}, \mathrm{z}$ \\
\hline \multirow{6}{*}{ QUE:DPE-I:ANT } & $\mathrm{O}(1)-\mathrm{H}(1 \mathrm{O}) \cdots \mathrm{N}(2)$ & 0.98 & $2.692(2)$ & 1.72 & 168 & $\mathrm{x},-1+\mathrm{y}, 1+\mathrm{z}$ \\
\hline & $\mathrm{O}(4)-\mathrm{H}(4 \mathrm{O}) \cdots \mathrm{O}(3)$ & 0.98 & $2.731(2)$ & 1.88 & $143^{\prime}$ & $x, 1-y, 2-z$ \\
\hline & $\mathrm{O}(5)-\mathrm{H}(5 \mathrm{O}) \cdots \mathrm{N}(1)$ & 0.98 & $2.692(2)$ & 1.71 & 172 & - \\
\hline & $\mathrm{O}(6)-\mathrm{H}(6 \mathrm{O}) \cdots \mathrm{O}(1)$ & 0.98 & $2.872(2)$ & 2.18 & $126^{\prime}$ & $1-\mathrm{x},-\mathrm{y}, 1-\mathrm{z}$ \\
\hline & $\mathrm{C}(2)-\mathrm{H}(2) \cdots \mathrm{O}(6)$ & 1.08 & $3.138(3)$ & 2.28 & 135 & $1-x,-y, 1-z$ \\
\hline & $\mathrm{C}(31)-\mathrm{H}(31) \cdots \mathrm{O}(4)$ & 1.08 & $3.471(3)$ & 2.48 & 152 & - \\
\hline
\end{tabular}


Table S2 Crystallographic tables for the experimental structures

\begin{tabular}{|c|c|c|c|c|c|c|c|}
\hline Compound & $\begin{array}{c}\text { QUE:TMP } \\
\text { Form I }\end{array}$ & $\begin{array}{c}\text { QUE:TMP } \\
\text { Form II }\end{array}$ & $\begin{array}{c}\text { QUE:TMP } \\
\text { Form II }\end{array}$ & $\begin{array}{c}\text { QUE:TMP } \\
\text { Form IV }\end{array}$ & $\begin{array}{c}\text { QUE:44BP } \\
\text { Form I }\end{array}$ & $\begin{array}{c}\text { QUE:44BP } \\
\text { Form II }\end{array}$ & $\begin{array}{c}\text { QUE:44BP } \\
\text { Form III }\end{array}$ \\
\hline CCDC No. & 1035762 & 1035763 & 1035765 & 1035764 & 1035766 & 1035767 & 1035768 \\
\hline Molecular formula & $\mathrm{C}_{39} \mathrm{H}_{46} \mathrm{~N}_{6} \mathrm{O}_{7}$ & $\mathrm{C}_{31} \mathrm{H}_{34} \mathrm{~N}_{4} \mathrm{O}_{7}$ & $\mathrm{C}_{27} \mathrm{H}_{30} \mathrm{~N}_{2} \mathrm{O}_{9}$ & $\mathrm{C}_{27} \mathrm{H}_{30} \mathrm{~N}_{2} \mathrm{O}_{8}$ & $\mathrm{C}_{29} \mathrm{H}_{26} \mathrm{~N}_{2} \mathrm{O}_{9}$ & $\mathrm{C}_{29} \mathrm{H}_{26} \mathrm{~N}_{2} \mathrm{O}_{8}$ & $\mathrm{C}_{26} \mathrm{H}_{22} \mathrm{~N}_{2} \mathrm{O}_{8}$ \\
\hline Formula weight & 710.82 & 574.62 & 526.53 & 510.53 & 546.52 & 530.52 & 536.51 \\
\hline Crystal system & Monoclinic & Monoclinic & Triclinic & Triclinic & Triclinic & Triclinic & Triclinic \\
\hline Space group & $P \mathrm{c}$ & $P 2_{1} / \mathrm{c}$ & $P 1$ & $P 1$ & $P 1$ & $P 1$ & $P 1$ \\
\hline$a(\AA)$ & $9.0760(13)$ & $15.831(2)$ & $9.3506(7)$ & $9.3202(10)$ & $9.8334(7)$ & $9.806(2)$ & $9.844(5)$ \\
\hline$b(\AA)$ & 8.9616(13) & $9.3122(13)$ & $10.9120(8)$ & $10.7538(12)$ & $10.7098(8)$ & $10.778(2)$ & $10.636(6)$ \\
\hline$c(\stackrel{\AA}{)})$ & $22.501(3)$ & $20.211(3)$ & 13.002(1) & $12.9869(14)$ & $13.0790(9)$ & 13.055(3) & $13.071(7)$ \\
\hline$\alpha\left(^{\circ}\right)$ & 90 & 90 & $76.798(5)$ & $79.471(6)$ & $102.815(7)$ & $102.833(7)$ & $98.324(7)$ \\
\hline$\beta\left(^{\circ}\right)$ & $93.554(5)$ & $104.415(7)$ & $84.048(6)$ & $85.133(6)$ & $95.031(7)$ & $94.525(7)$ & $94.601(7)$ \\
\hline$\gamma\left(0^{\circ}\right)$ & 90 & 90 & $81.580(6)$ & $82.377(6)$ & $106.846(7)$ & $106.943(7)$ & $107.947(8)$ \\
\hline$V\left(\AA^{3}\right)$ & $1826.6(4)$ & $2885.7(7)$ & $1274.26(17)$ & $1266.0(2)$ & $1268.30(17)$ & $1271.6(5)$ & $1276.9(12)$ \\
\hline pcalc $\left(\mathrm{g} / \mathrm{cm}^{3}\right)$ & 1.292 & 1.323 & 1.372 & 1.339 & 1.431 & 1.365 & 1.395 \\
\hline$F(000)$ & 756 & 1216 & 556 & 540 & 572 & 540 & 558 \\
\hline$\mu_{n}\left(\mathrm{~mm}^{-1}\right)$ & 0.090 & 0.095 & 0.104 & 0.099 & 0.107 & 0.101 & 0.101 \\
\hline$T(K)$ & $150 \mathrm{~K}$ & $150 \mathrm{~K}$ & $150 \mathrm{~K}$ & $150 \mathrm{~K}$ & $150 \mathrm{~K}$ & $150 \mathrm{~K}$ & $150 \mathrm{~K}$ \\
\hline$\lambda(\stackrel{\AA}{)}$ & 0.71073 & 0.71073 & 0.71073 & 0.71073 & 0.71073 & 0.71073 & 0.71073 \\
\hline Reflns. collected & 18413 & 22578 & 13650 & 13412 & 13454 & 10628 & 13246 \\
\hline Unique reflns. & 8351 & 6558 & 5832 & 5779 & 5791 & 4943 & 5767 \\
\hline Completeness (\%) & 99.2 & 99.2 & 99.9 & 99.7 & 99.7 & 99.3 & 99.0 \\
\hline $\boldsymbol{R}_{\text {int }}$ & 0.062 & 0.077 & 0.034 & 0.030 & 0.016 & 0.037 & 0.022 \\
\hline$R_{I}\left(F^{2}\right)$ & 0.0579 & 0.0628 & 0.0523 & 0.0698 & 0.0400 & 0.0748 & 0.0427 \\
\hline$w R_{2}\left(F^{2}\right)$ & 0.1304 & 0.1626 & 0.1587 & 0.2016 & 0.1097 & 0.2137 & 0.1137 \\
\hline Goodness-of-fit & 1.04 & 0.96 & 0.93 & 1.05 & 1.05 & 1.06 & 1.04 \\
\hline 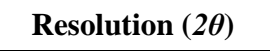 & 54 & 54 & 54 & 54 & 54 & 54 & 54 \\
\hline
\end{tabular}




\begin{tabular}{|c|c|c|c|c|c|c|c|}
\hline Compound & $\begin{array}{l}\text { QUE:44BP } \\
\text { Form IV }\end{array}$ & $\begin{array}{l}\text { QUE:DPE-I } \\
\text { Form I }\end{array}$ & $\begin{array}{l}\text { QUE:DPE-I } \\
\text { Form II }\end{array}$ & $\begin{array}{l}\text { QUE:DPE-I } \\
\text { Form III }\end{array}$ & $\begin{array}{l}\text { QUE:DPE-I } \\
\text { Form IV }\end{array}$ & $\begin{array}{l}\text { QUE:DPE-I } \\
\text { Form V }\end{array}$ & $\begin{array}{l}\text { QUE:DPE-II } \\
\text { Form I }\end{array}$ \\
\hline CCDC No. & 1035769 & 1035770 & 1035771 & 1035772 & 1035773 & 1035774 & 1035775 \\
\hline Molecular formula & $\mathrm{C}_{45} \mathrm{H}_{36} \mathrm{~N}_{6} \mathrm{O}_{8}$ & $\mathrm{C}_{31} \mathrm{H}_{28} \mathrm{~N}_{2} \mathrm{O}_{9}$ & $\mathrm{C}_{31} \mathrm{H}_{28} \mathrm{~N}_{2} \mathrm{O}_{8}$ & $\mathrm{C}_{30} \mathrm{H}_{26} \mathrm{~N}_{3} \mathrm{O}_{8}$ & $\mathrm{C}_{39} \mathrm{H}_{30} \mathrm{~N}_{4} \mathrm{O}_{7}$ & $\mathrm{C}_{27} \mathrm{H}_{20} \mathrm{~N}_{2} \mathrm{O}_{7}$ & $\mathrm{C}_{31} \mathrm{H}_{30} \mathrm{~N}_{2} \mathrm{O}_{8}$ \\
\hline Formula weight & 788.80 & 572.55 & 556.55 & 556.54 & 666.67 & 484.45 & 558.57 \\
\hline Crystal system & Triclinic & Triclinic & Triclinic & Triclinic & Monoclinic & Monoclinic & Triclinic \\
\hline Space group & $P 1$ & $P 1$ & $P 1$ & $P 1$ & $P 2_{1} / \mathrm{c}$ & $C 2 / \mathrm{c}$ & $P 1$ \\
\hline$a(\AA)$ & $10.0222(12)$ & 10.1899(9) & $10.0381(7)$ & $9.9867(15)$ & $18.0656(11)$ & $21.831(5)$ & $9.8782(8)$ \\
\hline$b(\stackrel{\AA}{)}$ & $12.8756(16)$ & $11.6532(11)$ & $11.5707(8)$ & $11.2949(12)$ & 11.6584(6) & 12.904(3) & $11.5881(10)$ \\
\hline$c(\stackrel{\AA}{)})$ & $15.0597(18)$ & $13.0214(12)$ & $13.0194(9)$ & $13.040(2)$ & $16.6610(1)$ & $15.876(4)$ & 13.041(1) \\
\hline$\alpha\left({ }^{\circ}\right)$ & $81.685(6)$ & $97.351(7)$ & $76.744(5)$ & $79.558(6)$ & 90 & 90 & $76.595(5)$ \\
\hline$\beta\left({ }^{\circ}\right)$ & $83.118(6)$ & $95.446(7)$ & $84.502(6)$ & $85.192(6)$ & $115.565(2)$ & $101.912(7)$ & $85.792(6)$ \\
\hline$\gamma\left({ }^{\circ}\right)$ & $76.273(5)$ & $115.237(8)$ & $65.573(5)$ & $66.082(5)$ & 90 & 90 & $67.035(5)$ \\
\hline$V\left(\AA^{3}\right)$ & $1860.6(4)$ & $1367.8(2)$ & $1340.13(17)$ & $1322.2(4)$ & $3165.5(3)$ & $4376.1(18)$ & $1336.8(2)$ \\
\hline pcalc $\left(\mathrm{g} / \mathrm{cm}^{3}\right)$ & 1.408 & 1.390 & 1.379 & 1.398 & 1.399 & 1.471 & 1.388 \\
\hline$F(000)$ & 824 & 600 & 584 & 582 & 1392 & 2016 & 588 \\
\hline$\mu\left(m m^{-1}\right)$ & 0.099 & 0.103 & 0.100 & 0.103 & 0.098 & 0.108 & 0.101 \\
\hline$T(K)$ & $150 \mathrm{~K}$ & $150 \mathrm{~K}$ & $150 \mathrm{~K}$ & $150 \mathrm{~K}$ & $150 \mathrm{~K}$ & $150 \mathrm{~K}$ & $150 \mathrm{~K}$ \\
\hline$\lambda(\AA)$ & 0.71073 & 0.71073 & 0.71073 & 0.71073 & 0.71073 & 0.71073 & 0.71073 \\
\hline Reflns. collected & 19338 & 14650 & 14382 & 13781 & 27138 & 18633 & 14292 \\
\hline Unique reflns. & 8502 & 6248 & 6106 & 6040 & 7197 & 5006 & 6112 \\
\hline Completeness (\%) & 99.7 & 99.7 & 99.8 & 99.7 & 99.1 & 99.5 & 99.8 \\
\hline$R_{\text {int }}$ & 0.031 & 0.035 & 0.034 & 0.052 & 0.038 & 0.071 & 0.036 \\
\hline$R_{I}\left(F^{2}\right)$ & 0.0452 & 0.0511 & 0.0566 & 0.0634 & 0.0468 & 0.0545 & 0.0544 \\
\hline$w R_{2}\left(F^{2}\right)$ & 0.1343 & 0.1323 & 0.1618 & 0.1776 & 0.1188 & 0.1497 & 0.1479 \\
\hline Goodness-of-fit & 0.88 & 1.02 & 1.03 & 1.02 & 1.04 & 1.05 & 1.04 \\
\hline 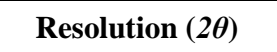 & 54 & 54 & 54 & 54 & 54 & 54 & 54 \\
\hline
\end{tabular}

\begin{tabular}{|l|l|l|l|l|l|l|l|} 
Compound & QUE:DPE-II & QUE:DPE-II & QUE:44AP & QUE:44AP & QUE:PHE & QUE:PHE & QUE:PHE \\
\hline
\end{tabular}




\begin{tabular}{|c|c|c|c|c|c|c|c|}
\hline & Form II & Form III & Form I & Form II & Form I & Form II & Form III \\
\hline CCDC No. & 1035776 & 1035777 & 1035778 & 1035751 & 1035752 & 1035753 & 1035754 \\
\hline Molecular formula & $\mathrm{C}_{39} \mathrm{H}_{34} \mathrm{~N}_{4} \mathrm{O}_{7}$ & $\mathrm{C}_{27} \mathrm{H}_{22} \mathrm{~N}_{2} \mathrm{O}_{7}$ & $\mathrm{C}_{29} \mathrm{H}_{26} \mathrm{~N}_{4} \mathrm{O}_{8}$ & $\mathrm{C}_{25} \mathrm{H}_{18} \mathrm{~N}_{4} \mathrm{O}_{7}$ & $\mathrm{C}_{31} \mathrm{H}_{26} \mathrm{~N}_{2} \mathrm{O}_{9}$ & $\mathrm{C}_{33} \mathrm{H}_{22} \mathrm{~N}_{3} \mathrm{O}_{7}$ & $\mathrm{C}_{58} \mathrm{H}_{42} \mathrm{~N}_{7} \mathrm{O}_{8}$ \\
\hline Formula weight & 670.70 & 486.47 & 558.54 & 486.43 & 570.54 & 572.54 & 964.99 \\
\hline Crystal system & Monoclinic & Monoclinic & Triclinic & Monoclinic & Triclinic & Triclinic & Triclinic \\
\hline Space group & $P 2_{1 / 1} / \mathrm{c}$ & $P 2_{1 / \mathrm{c}}$ & $P 1$ & $C 2 / \mathrm{c}$ & $P 1$ & $P 1$ & $P 1$ \\
\hline$a(\AA)$ & $20.028(3)$ & $13.3624(4)$ & $10.0918(17)$ & $21.647(3)$ & $8.1859(13)$ & $8.416(3)$ & $9.9945(3)$ \\
\hline$b(\stackrel{\cap}{)}$ & $10.5025(13)$ & $10.5540(4)$ & $11.4244(19)$ & $12.8623(16)$ & $12.787(2)$ & $12.297(4)$ & $13.4346(4)$ \\
\hline$c(\stackrel{\AA}{)})$ & $16.5786(18)$ & $32.5542(10)$ & 13.052(2) & $15.712(2)$ & 13.173(2) & 13.111(4) & $17.8220(5)$ \\
\hline$\alpha\left({ }^{\circ}\right)$ & 90 & 90 & 98.573(7) & 90 & $83.634(6)$ & $87.100(6)$ & $81.016(1)$ \\
\hline$\beta\left(^{\circ}\right)$ & $108.851(4)$ & $95.517(3)$ & $95.190(7)$ & $101.543(7)$ & $83.848(6)$ & $84.103(6)$ & $78.335(1)$ \\
\hline$\gamma\left(\left(^{\circ}\right)\right.$ & 90 & 90 & $115.078(8)$ & 90 & $73.246(5)$ & $73.503(5)$ & $86.046(1)$ \\
\hline$V\left(\AA^{3}\right)$ & $3300.2(7)$ & $4569.8(3)$ & $1327.7(4)$ & $4286.2(10)$ & 1308.1(4) & $1293.8(7)$ & $2313.09(12)$ \\
\hline pcalc $\left(\mathrm{g} / \mathrm{cm}^{3}\right)$ & 1.350 & 1.414 & 1.397 & 1.508 & 1.449 & 1.470 & 1.385 \\
\hline$F(000)$ & 1408 & 2032 & 584 & 2016 & 596 & 594 & 1006 \\
\hline$\mu\left(m^{-1}\right)$ & 0.094 & 0.103 & 0.104 & 0.113 & 0.108 & 0.105 & 0.094 \\
\hline$T(K)$ & $150 \mathrm{~K}$ & $150 \mathrm{~K}$ & $150 \mathrm{~K}$ & $150 \mathrm{~K}$ & $150 \mathrm{~K}$ & $150 \mathrm{~K}$ & $150 \mathrm{~K}$ \\
\hline$\lambda(\stackrel{\circ}{)}$ & 0.71073 & 0.71073 & 0.71073 & 0.71073 & 0.71073 & 0.71073 & 0.71073 \\
\hline Reflns. collected & 36152 & 43116 & 11363 & 24298 & 12319 & 10572 & 29578 \\
\hline Unique reflns. & 7537 & 10476 & 5197 & 4173 & 5126 & 5028 & 10501 \\
\hline Completeness (\%) & 99.4 & 99.9 & 99.6 & 99.0 & 99.8 & 98.8 & 98.6 \\
\hline $\boldsymbol{R}_{\text {int }}$ & 0.053 & 0.050 & 0.074 & 0.051 & 0.032 & 0.128 & 0.033 \\
\hline$R_{I}\left(F^{2}\right)$ & 0.0450 & 0.0530 & 0.0898 & 0.0517 & 0.0544 & 0.0845 & 0.0456 \\
\hline$w R_{2}\left(F^{2}\right)$ & 0.1133 & 0.1499 & 0.2647 & 0.1362 & 0.1435 & 0.2156 & 0.1203 \\
\hline Goodness-of-fit & 1.04 & 1.02 & 1.17 & 0.89 & 1.08 & 0.99 & 1.03 \\
\hline 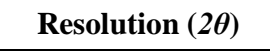 & 54 & 54 & 54 & 54 & 54 & 54 & 54 \\
\hline Compound & $\begin{array}{l}\text { QUE:PHE } \\
\text { Form IV }\end{array}$ & $\begin{array}{c}\text { QUE:PHE } \\
\text { Form V }\end{array}$ & $\begin{array}{c}\text { QUE:44BP } \\
\text { 22TP }\end{array}$ & $\begin{array}{c}\text { QUE:44BP } \\
\text { TTF }\end{array}$ & $\begin{array}{c}\text { QUE:DPE-I } \\
\text { 22TP } \\
\end{array}$ & $\begin{array}{c}\text { QUE:DPE-I } \\
\text { PYR } \\
\end{array}$ & $\begin{array}{c}\text { QUE:DPE-I } \\
\text { ANT }\end{array}$ \\
\hline CCDC No. & 1035755 & 1035756 & 1035757 & 1035758 & 1035759 & 1035760 & 1035761 \\
\hline
\end{tabular}




\begin{tabular}{|c|c|c|c|c|c|c|c|}
\hline Molecular formula & $\mathrm{C}_{33} \mathrm{H}_{24} \mathrm{~N}_{3} \mathrm{O}_{8}$ & $\mathrm{C}_{33} \mathrm{H}_{22} \mathrm{~N}_{3} \mathrm{O}_{7}$ & $\mathrm{C}_{29} \mathrm{H}_{21} \mathrm{~N}_{2} \mathrm{O}_{7} \mathrm{~S}$ & $\mathrm{C}_{69} \mathrm{H}_{50} \mathrm{~N}_{6} \mathrm{O}_{14} \mathrm{~S}_{6}$ & $\mathrm{C}_{31} \mathrm{H}_{23} \mathrm{~N}_{2} \mathrm{O}_{7} \mathrm{~S}$ & $\mathrm{C}_{90} \mathrm{H}_{63} \mathrm{~N}_{6} \mathrm{O}_{14}$ & $\mathrm{C}_{34} \mathrm{H}_{24} \mathrm{~N}_{2} \mathrm{O}_{7}$ \\
\hline Formula weight & 590.56 & 572.54 & 541.55 & 1379.57 & 567.59 & 1452.46 & 572.55 \\
\hline Crystal system & Triclinic & Triclinic & Triclinic & Triclinic & Triclinic & Triclinic & Triclinic \\
\hline Space group & $P 1$ & $P 1$ & $P 1$ & $P 1$ & $P 1$ & $P 1$ & $P 1$ \\
\hline$a(\AA)$ & $9.4800(7)$ & 4.9830 & $9.8066(9)$ & $12.547(2)$ & 9.974 & 12.664 & 10.5919(6) \\
\hline$b(\stackrel{\AA}{)}$ & $11.2614(9)$ & 15.3810 & $10.4874(10)$ & 13.299(2) & 11.421 & 13.235 & $11.6067(6)$ \\
\hline$c(\AA)$ & $13.5129(10)$ & 18.1420 & 13.0073(12) & $18.490(3)$ & 13.009 & 22.191 & 11.6919(7) \\
\hline$\alpha\left({ }^{\circ}\right)$ & $104.785(4)$ & 112.210 & 97.993(7) & $94.246(7)$ & 78.510 & 104.030 & $85.133(4)$ \\
\hline$\beta\left(^{\circ}\right)$ & $108.855(4)$ & 94.990 & 94.646(7) & $90.204(6)$ & 85.220 & 96.590 & $72.938(3)$ \\
\hline$\gamma\left({ }^{\circ}\right)$ & $90.687(4)$ & 94.920 & $108.150(8)$ & $98.776(7)$ & 66.390 & 104040 & $75.632(3)$ \\
\hline$V\left(\AA^{3}\right)$ & $1312.95(18)$ & 1271.766 & $1247.8(2)$ & $3040.5(8)$ & 1330.627 & 3440.593 & 1331.01(13) \\
\hline $\operatorname{\rho calc}\left(\mathrm{g} / \mathrm{cm}^{3}\right)$ & 1.489 & 1.495 & 1.441 & 1.507 & 1.379 & 1.402 & 1.429 \\
\hline$F(000)$ & 610 & 594 & 562 & 1428 & 572 & 1514 & 596 \\
\hline$\mu\left(\mathrm{mm}^{-1}\right)$ & 0.108 & 0.107 & 0.184 & 0.302 & 0.174 & 0.096 & 0.101 \\
\hline$T(K)$ & $150 \mathrm{~K}$ & $150 \mathrm{~K}$ & $150 \mathrm{~K}$ & $150 \mathrm{~K}$ & $150 \mathrm{~K}$ & $150 \mathrm{~K}$ & $150 \mathrm{~K}$ \\
\hline$\lambda(\stackrel{\cap}{)}$ & 0.71073 & 0.71073 & 0.71073 & 0.71073 & 0.71073 & 0.71073 & 0.71073 \\
\hline Reflns. collected & 14918 & 13154 & 13323 & 31942 & 14146 & 35694 & 20837 \\
\hline Unique reflns. & 5100 & 5827 & 5709 & 13902 & 6078 & 15699 & 5395 \\
\hline Completeness (\%) & 99.0 & 99.6 & 99.8 & 99.6 & 99.8 & 99.5 & 99.0 \\
\hline $\boldsymbol{R}_{\text {int }}$ & 0.042 & 0.066 & 0.031 & 0.064 & 0.060 & 0.096 & 0.050 \\
\hline$R_{I}\left(F^{2}\right)$ & 0.0456 & 0.0614 & 0.0526 & 0.0666 & 0.0679 & 0.0804 & 0.0501 \\
\hline$w R_{2}\left(F^{2}\right)$ & 0.1226 & 0.1549 & 0.1421 & 0.1877 & 0.2020 & 0.2131 & 0.1400 \\
\hline Goodness-of-fit & 0.99 & 0.97 & 1.06 & 1.03 & 1.04 & 1.02 & 1.04 \\
\hline Resolution $(2 \theta)$ & 52 & 54 & 54 & 54 & 54 & 54 & 52 \\
\hline
\end{tabular}

